\title{
Implementation of the Maximum Entropy Method for Analytic Continuation
}

\author{
Ryan Levy ${ }^{\mathrm{a}}$, J.P.F. LeBlanc ${ }^{\mathrm{a}}$, Emanuel Gull ${ }^{\mathrm{a}, *}$ \\ ${ }^{a}$ Department of Physics, University of Michigan, Ann Arbor, MI 48109, USA
}

\section{Abstract}

We present Maxent, a tool for performing analytic continuation of spectral functions using the maximum entropy method. The code operates on discrete imaginary axis datasets (values with uncertainties) and transforms this input to the real axis. The code works for imaginary time and Matsubara frequency data and implements the 'Legendre' representation of finite temperature Green's functions. It implements - a variety of kernels, default models, and grids for continuing bosonic, fermionic, anomalous, and other data. Our implementation is licensed under GPLv2 and extensively documented. This paper shows the use of the programs in detail.

Keywords: Maximum Entropy Method, Analytic Continuation

\section{PROGRAM SUMMARY}

Manuscript Title: Implementation of the Maximum Entropy Method for Analytic Continuation

Authors: Ryan Levy, J.P.F. LeBlanc, Emanuel Gull

Program Title: maxent

Journal Reference:

Catalogue identifier:

Licensing provisions: GPLv2

Programming language: $\mathrm{C}++$

Operating system: Tested on Linux and Mac OS X

RAM: $10 \mathrm{MB}-200 \mathrm{MB}$

Keywords: Maximum Entropy Method, Analytic

Continuation

Classification: 4.9

External routines/libraries: ALPSCore [1] 2], GSL, HDF5

Nature of problem: The analytic continuation of imaginary axis correlation functions to real frequency/time variables is an ill-posed problem which has an infinite number of solutions.

Solution method: The maximum entropy method obtains a possible solution that maximizes entropy, enforces sum rules, and otherwise produces 'smooth' curves. Our implementation allows for input in Matsubara frequencies, imaginary time, or a Legendre expansion. It implements a range of bosonic,

\footnotetext{
*Corresponding author.

E-mail address: egull@umich.edu
}

Preprint submitted to Computer Physics Communications fermionic and generalized kernels for normal and anomalous Green's functions, self-energies, and two-particle response functions.

Running time: $10 \mathrm{~s}-2 \mathrm{~h}$ per solution

[1] B. Bauer, et al., The ALPS project release 2.0: open source software for strongly correlated systems, J. Stat. Mech. Theor. Exp. 2011 (05) (2011) P05001. arXiv:1101.2646 doi:10.1088/17425468/2011/05/P05001.

[2] A. Gaenko, E. Gull, A. E. Antipov, L. Gamper, G. Carcassi, J. Paki, R. Levy, M. Dolfi, J. Greitemann, J.P.F. LeBlanc, Alpscore: Version 0.5.4doi: 10.5281/zenodo.50203.

\section{Introduction}

Analytic continuation of numerical data is a standard problem in condensed matter physics. It primarily appears when correlation functions of a many-body problem, computed in an imaginary time statistical mechanics formulation, need to be interpreted as response or spectral functions on the real axis. While imaginary time (or the Fourier transform, Matsubara frequency) correlation functions are naturally obtained in numerical 
simulations such as quantum Monte Carlo lattice [3] and impurity solvers [4-7], their real axis counterparts that correspond to response functions, which are measured in experiment, are not typically accessible to numerical techniques.

At the heart of this is that the continuation from the imaginary axis to the real axis is exceptionally ill conditioned, such that small fluctuations of the input data (either from statistical Monte Carlo noise or a truncation of the accuracy to finite precision numbers) lead to large fluctuations of the output data, rendering any direct transformations useless in practice. Several alternatives have been proposed, among them the construction of rational polynomial functions (Padé approximants) [8-10], a constrained optimization procedure [11], a stochastic analytic continuation method [12], and a stochastic analytic inference method [13]. The standard method, however, is the so-called maximum entropy method (MEM) [14, 15], for which we provide an implementation in this paper. Our implementation, Maxent, is part of the ALPS applications [1, 16, 17] and makes use of the core ALPS libraries [2]. In the following, we will briefly review the formalism (referring the reader to Ref. [14] and the original literature for more details), introduce our implementation, and illustrate its usage with examples.

\section{Analytic Continuation}

\subsection{Analytic Continuation Formalism}

We start our considerations with the imaginary time Green's function $G(\tau)=-\left\langle c(\tau) c^{\dagger}(0)\right\rangle$, which is a continuous function for $0<\tau<\beta$, and is periodic for bosonic and anti-periodic for fermionic systems within $\tau \in[0, \beta]$. In this notation, $c$ denotes an annihilation operator, $c^{\dagger}$ a creation operator, and the time-dependence of the operator and its expectation value are to be interpreted in the usual sense [18]. Imaginary time Green's functions of this type are the fundamental objects that most QMC methods produce as a simulation output. The Green's function in $\tau$ can be related to the Green's function on the imaginary frequency axis through a Fourier transform

$$
G\left(i \omega_{n}\right)=\int_{0}^{\beta} e^{i \omega_{n} \tau} G(\tau) .
$$

The 'Matsubara' frequencies $i \omega_{n}$ come from poles of the distribution functions and are defined as $i \omega_{n}=2 \pi\left(n+\frac{1}{2}\right) / \beta$ for fermionic and $i \omega_{n}=2 \pi n / \beta$ for bosonic operators.

For the rest of the article, we assume that these Green's functions are not known to arbitrary precision. Rather, we work with a truncation of the Green's function to $N$ components, which are obtained by averaging a set of $M$ estimates for each component, $G_{n}^{(i)}$, that are independent and Gaussian distributed so that if there are $M$ samples for each $n$, the estimate for the Green's function is given by

$$
G_{n}=\frac{1}{M} \sum_{j=1}^{M} G_{n}^{(j)}
$$

Different components $n$ and $m$ of the Green's function may be correlated. This is encapsulated in the covariance matrix $C_{n m}$, which is estimated as

$$
C_{n m}=\frac{1}{M(M-1)} \sum_{j=1}^{M}\left(G_{n}-G_{n}^{(j)}\right)\left(G_{m}-G_{m}^{(j)}\right) .
$$

In the case of fermions, the Matsubara frequency Green's function $G\left(i \omega_{n}\right)$ and its imaginary time counterpart $G(\tau)$ are related to a real frequency Green's function $G(\omega)$ via

$$
\begin{aligned}
G\left(i \omega_{n}\right) & =\frac{-1}{\pi} \int_{-\infty}^{\infty} \frac{d \omega \operatorname{Im}[G(\omega)]}{i \omega_{n}-\omega} \\
G\left(\tau_{n}\right) & =\frac{1}{\pi} \int_{-\infty}^{\infty} \frac{d \omega \operatorname{Im}[G(\omega)] e^{-\tau_{n} \omega}}{1+e^{-\beta \omega}}
\end{aligned}
$$

where $\tau$ has been discretized in some manner to $N$ points. The imaginary part of the Green's function that appears in the numerators of Eqs. (4) and (5) defines the spectral function

$$
A(\omega)=-\frac{1}{\pi} \operatorname{Im}[G(\omega)] .
$$

Obtaining $G(\omega)$ and $A(\omega)$ in addition to related quantities for bosonic and other response functions, as well as self-energies, is the main purpose of this paper. 
We can formulate Eq. 5 as

$$
\begin{aligned}
G_{n}=G\left(\tau_{n}\right) & =\int_{-\infty}^{\infty} d \omega A(\omega) K_{n}(\omega), \\
K_{n}(\omega) & =K\left(\tau_{n}, \omega\right)=-\frac{e^{-\tau_{n} \omega}}{1+e^{-\omega \beta}},
\end{aligned}
$$

where $K_{n}$ is the 'kernel' of the analytic continuation, here for a transformation of a fermionic Green's function from imaginary time to real frequencies. Kernels for other distribution functions and imaginary axis representations are listed in Sec. 3.3, Tables 3 and 4 .

Given a candidate spectral function $A(\omega)$ on the real axis and the associated kernel, the imaginary axis Green's function can be evaluated using Eq. (7) to create an estimate $\bar{G}_{n}$, a process known as a back-continuation. To calculate the consistency of a spectral function $A(\omega)$ with the imaginary axis data $G_{n}$, one can define a "goodness of fit" quantity $\chi^{2}$

$$
\chi^{2}=\sum_{n, m}^{M}\left(\bar{G}_{n}-G_{n}\right)^{*} C_{n m}^{-1}\left(\bar{G}_{m}-G_{m}\right),
$$

where $C_{n m}$ is defined in Eq. (3). Consistency of $A$ with $G_{n}$ within errors given by $C_{n m}$ is achieved for $\chi^{2} \sim M$. If the input data is uncorrelated then only the diagonal elements of the covariance matrix are non-zero, in which case $\chi^{2}$ takes the form

$$
\chi^{2}=\sum_{n}^{M} \frac{\left(\bar{G}_{n}-G_{n}\right)^{2}}{\sigma_{n}^{2}}
$$

where $\sigma_{n}$ is the standard error in $G_{n}$.

\subsection{Inversion of the Kernel}

To computationally solve for $A(\omega)$ in Eq. (7), the simplest method to employ is a least squares fitting routine, which attempts to minimize a functional $Q=\chi^{2}$ with $\chi^{2}$ described in Eq. (9). While back-continuation is a straightforward procedure that gives a unique result, the inversion of Eq. (7) is ill conditioned, i.e. there are many solutions $A$ that satisfy $G=K A$ to within the uncertainty given by $C_{n m}$.

\subsection{Maximum Entropy Method}

Instead of least-squares fitting, analytic continuation algorithms impose additional criteria on $A$, such as smoothness [11, in order to reduce the space of acceptable solutions. In the maximum entropy method an "entropy" term, $S$, is also considered to help regularize the solutions, such that the functional to minimize becomes

$$
Q=\frac{1}{2} \chi^{2}-\alpha S[A]
$$

where the factor of $1 / 2$ is added for mathematical convenience and we have introduced a Lagrange multiplier $\alpha>0$ here, in order to control the competition between $\chi^{2}$ and $S$.

Shannon entropy [19] is used in order to minimize spurious correlations between data [20]; this restricts its application to cases where the resulting spectral function is finite and positive (or can be transformed to be), so it can be treated as a probability density [14].

Only changes in entropy are meaningful, and therefore entropy is defined with respect to a reference spectral function, the default model $d(\omega)$. This function eliminates the dependence on the choice of frequency grid or other fitting parameters. The entropy term is given by

$$
S[A]=-\int d \omega A(\omega) \ln \left[\frac{A(\omega)}{d(\omega)}\right] .
$$

When using a default model, considering a range of $\alpha$ values becomes favorable, for example with: $\alpha \gg 1$, we obtain the default model, which is an attempt to ensure a sensible solution that is ideally independent of the input data. For $\alpha \ll 1$, we again recover the least squares fit. While the default model provides a starting estimate $A(\omega)$, the final result is often insensitive to the choice of default model.

This formalism can also be motivated in an equilibrium statistical mechanics formalism, where the free energy $F$ will be minimized. Since $F=U-T S$ there is interplay between internal energy $U$ and entropy $S$. $Q$ can therefore be thought of as a quasi-free-energy where the parameter $\alpha$, much like $T$, contributes to the interplay between $U$ and $S$, where $U$ is represented by $\frac{1}{2} \chi^{2}$ 


\subsection{Algorithms for the Maximum Entropy Method}

The $Q$ functional of Eq. (11) requires one to systematically determine the Lagrange multiplier, $\alpha$, and spectral function, $A$. Ref. [14] lists two methods of determining a spectral function. The first, classic maxent (in the terminology in Ref. [14]), uses Bayesian inference to determine a spectral function that maximizes the function's posterior probability. The other method, Bryan's method [15], instead takes all spectral functions found for a given range of $\alpha$ values and averages them by their posterior probability [15]. Since classic maxent is a subset of Bryan's method, this allows for both to be computed within the same run of a program.

Bryan also provides an algorithm for reducing the search space of the kernel, by use of single value decomposition (SVD). For all of the kernels implemented in Maxent, the eigenvalues of the kernel drastically drop off in magnitude providing a search space generally between 8-20 dimensions. Additonally, Bryan gives the formalism to minimize $Q$ using a Levenberg-Marquardt routine [21]. The code Maxent implements Bryan's algorithm as described in Ref. [14, 15].

Other algorithms to find solutions using the MEM exist, including those that interpret the entropy curve [12] or finding a kink in a double-log plot of $\chi^{2}$ [22] in order to optimize $\alpha$. The MEM has also been shown to be a special limit of the stochastic analytic continuation algorithm [22].

\subsection{Error Estimates}

In addition to performing the inversion, Maxent is able to obtain systematic error bars that quantify variation in the classic maxent solution. In Ref. [14, Jarrell and Gubernatis give an analysis of the curvature of the objective $Q$. They provide a second order approximation to the covariance matrix $\left\langle\delta A_{i} \delta A_{j}\right\rangle$ of the most probable spectral function. Using this covariance matrix, we have designed a routine to rotate $\sqrt{A}$ into a diagonal basis, bootstrap within that basis, and rotate back to determine the error bar on the spectral function $A$.
The fitting routine itself and the dependence on the default model can be interpreted as an alternate source of error. This should give some semblance to the systematic error in the fitting routine, however it underestimates the error, except for data that has a strong default model dependence where the dominant error becomes the choice of default model. To aid in understanding the default model dependence, Maxent can run sequential calculations given choices of default model.

\section{Implementation}

All Maxent program options can be listed with a standard --help option, and these are elaborated upon in the code documentation. We note the most common user control parameters and pertinent details in this section.

\subsection{Input Basis}

There are three choices for input basis: imaginary time, frequency, and Legendre. Imaginary time consists of a real Green's function on $\tau \in$ $[0, \beta]$. The frequency basis is a complex valued Green's function consisting of Masubara frequencies defined in the usual way.

When the spectral function is symmetric about the Fermi level (generally taken to be $\omega=0$ ), the Green's function is known to have particle-hole symmetry. For fermionic Green's functions in the Matsubara basis, this corresponds to a vanishing real part, or vanishing imaginary part for bosonic Matsubara data. This allows us to only consider the real/imaginary part of the kernel, as shown in Table 3 ,

Maxent supports, in addition to Green's functions, self-energies [23] and correlation functions. The self-energies and correlation functions do not generally conform to the same symmetries and normalization properties as Green's functions, so Maxent multiplies by the proper normalization so that the effective spectral function is entirely positive. Normalization can also be provided by the user, for cases where the total spectral weight is not unity. Once the effective spectral function 
has been calculated, Maxent reverses the normalization in the output.

This code also implements a fermionic Legendre representation of the Green's function. As formulated by Boehnke, et al. [24, a Green's function can be expanded in the basis of Legendre polynomials given by the transforms

$$
\begin{array}{r}
G(\tau)=\sum_{\ell} \frac{\sqrt{2 \ell+1}}{\beta} P_{\ell}[x(\tau)] G_{\ell}, \\
G_{\ell}=\sqrt{2 \ell+1} \int_{0}^{\beta} d \tau P_{\ell}[x(\tau)] G(\tau),
\end{array}
$$

where $P_{\ell}$ is the $\ell$ th Legendre polynomial and $x(\tau)=2 \tau / \beta-1 \in[-1,1]$. For particle-hole symmetric Legendre data, odd $\ell$ values will vanish.

Representing a Green's function in an orthogonal polynomial basis, specifically the Legendre basis, has two major benefits over the time or frequency representation. Because of the density of information, the storage size for a Green's function is significantly reduced [25]. This is advantageous when storing Green's function objects for large systems. The other benefit is that the kernel is generally at most on the order of about $40 \times N_{\omega}$. For a small number of $\omega$ grid points $N_{\omega}$, Maxent will perform considerably faster compared to other data representations. This becomes important for general many-body problems where a Green's function has another index dependence, e.g. orbital number or momentum $\mathbf{k}$, in addition to $\tau$ or $i \omega_{n}$. In this case, one must perform separate Maxent runs for each index point.

\subsection{Default Models and Grids}

To generate the real frequency grid of the output spectral function, a user defined default model can be used (where the grid is taken from the defined points of the model), or a default model and grid can be generated with program options. The default models and grids are defined explicitly in Tables 1 and 2 in terms of their default and user required parameters, all of which are mutable via program options. The spectral function is then evaluated only on these grid points.

\subsection{Kernels}

Maxent implements several common kernel choices, for fermionic, bosonic, and anomalous Green's functions with and without particle-hole symmetry. These are shown in Tables 3 and 4 . After choosing the input basis and default model (with $\omega$ grid), the kernel is automatically set up. There is an additional problem with the bosonic kernel, where the kernel is not only singular for $\omega=0$ and $n=0$ but the spectral function is negative below the Fermi level. To overcome this, Maxent uses the kernel

$$
K_{n}(\omega)=\frac{\omega}{i \omega_{n}+\omega}
$$

which relates to the effective function $B(\omega)=$ $\chi^{\prime \prime}(\omega) / \omega$; after the calculation of $B(\omega)$, Maxent also produces $\chi^{\prime \prime}(\omega)=\omega B(\omega)$ [14].

For a Green's function represented by $G_{\ell}$ in the Legendre basis, the representation of Eq. (7) can be written with a kernel

$$
K_{\ell}(\omega) \equiv-\sqrt{2 \ell+1} \int_{-1}^{1} d x \frac{e^{-(1+x) \beta \omega / 2}}{1+e^{-\beta \omega}} P_{\ell}(x) .
$$

Maxent uses GSL to integrate the Legendre kernel [26].

\begin{tabular}{|c|c|c|}
\hline Dataspace & Kernel Name & Kernel \\
\hline \hline \multirow{2}{*}{ Frequency } & Fermionic & $\frac{1}{i \omega_{n}-\omega}$ \\
\cline { 2 - 3 } Without PH & Bosonic & $\frac{\omega}{i \omega_{n}+\omega}$ \\
\cline { 2 - 3 } & Anomalous & $\frac{-\omega}{i \omega_{n}-\omega}$ \\
\hline \multirow{2}{*}{ Frequency } & Fermionic & $\frac{\omega_{n}}{\omega_{n}^{2}+\omega^{2}}$ \\
\cline { 2 - 3 } With PH & Bosonic & $\frac{\omega^{2}}{\omega_{n}^{2}+\omega^{2}}$ \\
\cline { 2 - 3 } & Anomalous & $\frac{\omega^{2}}{\omega_{n}^{2}+\omega^{2}}$ \\
\hline
\end{tabular}

Table 3: Kernels in Matsubara frequency 


\begin{tabular}{|c|c|}
\hline Kernel Name & Kernel \\
\hline \hline Fermionic & $-\frac{e^{-\tau \omega}}{1+e^{-\omega \beta}}$ \\
\hline Bosonic & $\frac{1}{2} \omega \frac{\left[e^{-\omega \tau}+e^{-\omega(\beta-\tau)}\right]}{1-e^{-\omega \beta}}$ \\
\hline TZero & $-e^{-\omega \tau}$ \\
\hline
\end{tabular}

Table 4: Kernels in imaginary time

\section{Examples}

We provide four detailed examples for the different data representations available to Maxent.

In order to provide physically motivated examples, we include example data generated for a relevant model of correlated systems, the Hubbard model on a square lattice in 2-dimensions, which has been the topic of rigorous numerical benchmarking [27].

The Hamiltonian of the interacting Hubbard model can be written as

$$
H=-\sum_{<i j>\sigma} t\left(c_{i \sigma}^{\dagger} c_{j \sigma}+c_{j \sigma}^{\dagger} c_{i \sigma}\right)+U \sum_{i} n_{i \uparrow} n_{i \downarrow}
$$

where $c, c^{\dagger}$ are the annihilation/creation operators and the sum is over nearest neighbor sites $i, j$ with spin $\sigma$ and hopping amplitude $t$. Solutions to the Hubbard model using dynamical mean field theory (DMFT) will undergo a transition to a Mott insulator upon increasing the ratio of $U / t$ at halffilling [28 30].

\subsection{Fermionic Green's Function}

Here we present a walk-through of the noninteracting Hubbard Model of Eq. (17) at $U / t=$ $0, \beta t=8$. The problem can be solved analytically for $U=0$. One can then generate a local Green's function in Matsubara frequencies. All example data unless otherwise noted was calculated at half-filling, and thus only the imaginary part is needed as input.

For this example, the frequency space input data is in the file "G_im," which has columns of the form " $\left.\left.i \omega_{n}\right\lrcorner \operatorname{Im}\left[G_{n}\right]\right\lrcorner \sigma_{n}$ ", where the set of $\sigma_{n}$ are simulated errors chosen to be a fixed small value to avoid an over constrained fitting routine. The parameter input file provided to Maxent is:

\begin{tabular}{|c|c|}
\hline \multicolumn{2}{|c|}{ Param File in.param } \\
\hline $\mathrm{BET} A=8$ & \#inverse temperature \\
\hline $\mathrm{NDAT}=1024$ & \#num of data points \\
\hline NFREQ $=500$ & \#num of output \\
\hline \multicolumn{2}{|l|}{ frequencies } \\
\hline DATASPACE $=$ frequency & $\# G(i \omega)$ \\
\hline $\begin{array}{c}\text { KERNEL }=\text { fermionic } \\
\text { values }\end{array}$ & \#fermionic/bosonic \\
\hline \multicolumn{2}{|c|}{ PARTICLE_HOLE_SYMMETRY $=1 \quad \# O / 1$} \\
\hline $\begin{array}{c}\text { DATA="G_im" } \\
\text { file }\end{array}$ & \#location of data \\
\hline
\end{tabular}

Running maxent --help will list default parameters used for this simulation.

\subsubsection{Output Guide}

Maxent produces useful pieces of output during its calculations which are discussed in the example documents found in the folder "examples." The SVD of this data set reduces the kernel to a singular space of 8 vectors, which produces a most probable spectrum and Bayesian averaged spectrum with $60 \alpha$ values in the range $[0.01,20]$. The normalization of the spectral function varies from unity by no more than $3 \times 10^{-4}$ and when back-continued, the two spectra have an error no larger than $4 \times 10^{-4}$ - both of which are a sign of a successful calculation.

If the parameter TEXT_OUTPUT is set to true, Maxent will produce eight files described in Table 5. The main output is the spectral function obtained using Bryan's method, which is written to in.out.avspec.dat (given an input file named in.param - see Table 5) and is shown in Figure 1.

\subsection{Fermionic Self-Energy}

Using DMFT to solve a single site impurity problem on a square lattice, one can generate a local Green's function and self-energy for a metallic phase $(U / t=1)$ and an insulating phase $(U / t=10)$. Further explanation and datasets are available in the repository.

To activate the self-energy continuation in Maxent, the parameter SELF needs to be set to 


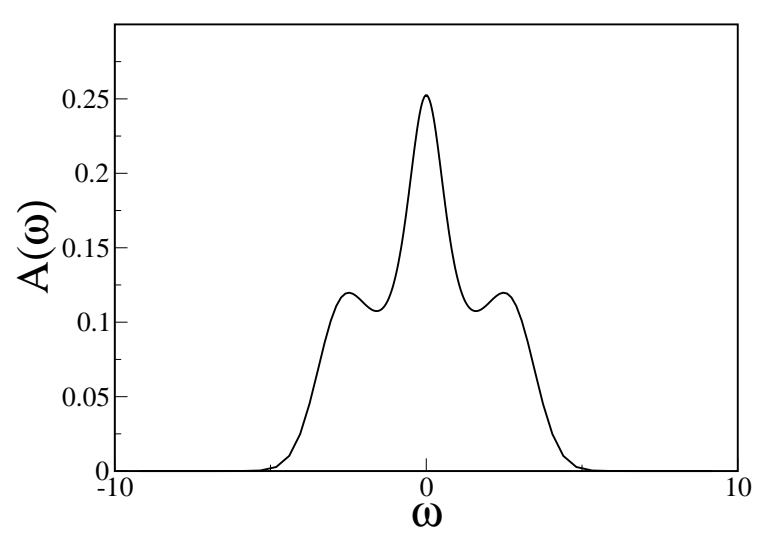

Figure 1: Spectral function from Maxent (Bryan's method) using data at $U / t=0, \beta / t=8$

true. This produces files in.out.avspec_self.dat and in.out.maxspec_self.dat which are the properly normalized self-energies for those respective functions. The self-energy output of Maxent is shown in Figure $2 a-b$.

\subsection{Legendre Representation}

In order to provide an example for the Legendre kernel in Maxent, a spectral function consisting of three Gaussian functions was calculated, back-continued into a fermionic imaginary time Green's function, and finally transformed into the Legendre basis. The Legendre representation of the data is shown in Figure 3 a. To use a Green's function in the Legendre basis, change the parameter DATASPACE to "Legendre" and keep KERNEL set to "fermionic". Using only the first 10 points, Maxent was able to produce an output shown in Figure 3b, and when back-continued has absolute error shown in the inset of Figure 3 a.

\subsection{Bosonic Green's Function}

The Hubbard model in Eq. (17) can also be solved for 2 particle correlation functions. We apply the dynamical cluster approximation on an 8site cluster to obtain the magnetic susceptibility, $\chi\left(i \omega_{n}\right)$, as a function of bosonic Matsubara frequencies for the two-dimensional Hubbard model at $U / t=6, n=0.9, \beta t=2$ at a scattering momentum of $Q=(\pi, \pi)$.

To perform Maxent on bosonic data, the parameter KERNEL must be set to "bosonic". Maxent will produce extra files in.out.avspec_bose.dat and in.out.maxspec_bose.dat which are the properly normalized $\chi^{\prime \prime}(\omega)$, where in the usual spectral output, in.out.avspec.dat for instance, will be $B(\omega)$. The results of both $\chi^{\prime \prime}(\omega)$ and $B(\omega)$ from Maxent are shown in Figure $4 a-b$.

\section{Acknowledgments}

This project was supported by the Simons Collaboration on the Many-Electron Problem. We gratefully acknowledge assistance from the ALPSCore community and Alex Gaenko.

[1] B. Bauer, L. D. Carr, H. G. Evertz, A. Feiguin, J. Freire, S. Fuchs, L. Gamper, J. Gukelberger, E. Gull, S. Guertler, A. Hehn, R. Igarashi, S. V. Isakov, D. Koop, P. N. Ma, P. Mates, H. Matsuo, O. Parcollet, G. Pawowski, J. D. Picon, L. Pollet, E. Santos, V. W. Scarola, U. Schollwöck, C. Silva, B. Surer, S. Todo, S. Trebst, M. Troyer, M. L. Wall, P. Werner, S. Wessel, The ALPS project release 2.0: open source software for strongly correlated systems, J. Stat. Mech. Theor. Exp. 2011 (05) (2011) P05001. arXiv:1101.2646, doi:10.1088/1742-5468/2011/ 05/P05001.

[2] A. Gaenko, E. Gull, A. E. Antipov, L. Gamper, G. Carcassi, J. Paki, R. Levy, M. Dolfi, J. Greitemann, J. P. F. LeBlanc, Alpscore: Version 0.5.4doi: 10.5281/zenodo.50203.

[3] R. Blankenbecler, D. J. Scalapino, R. L. Sugar, Monte carlo calculations of coupled boson-fermion systems. i. Phys. Rev. D 24 (8) (1981) 2278-2286. doi: 10.1103/PhysRevD.24.2278.

URL http://dx.doi.org:10.1103/PhysRevD.24. 2278

[4] A. N. Rubtsov, V. V. Savkin, A. I. Lichtenstein, Continuous-time quantum monte carlo method for fermions, Phys. Rev. B 72 (3) (2005) 035122. doi: 10.1103/PhysRevB.72.035122.

URL http://dx.doi.org/10.1103/PhysRevB.72. 035122

[5] P. Werner, A. Comanac, L. de' Medici, et al., Continuous-time solver for quantum impurity models, Phys. Rev. Lett. 97 (7) (2006) 076405. doi: 10.1103/PhysRevLett.97.076405.

URL http://dx.doi.org/10.1103/PhysRevLett. 97.076405

[6] E. Gull, P. Werner, O. Parcollet, M. Troyer, Continuous-time auxiliary-field monte carlo for quantum impurity models, EPL (Eu\begin{tabular}{lllll}
\hline rophysics Letters) & 82 & $(5)$ & $(2008)$ & 57003.
\end{tabular} doi:10.1209/0295-5075/82/57003.

URL http://stacks.iop.org/0295-5075/82/ 57003 
[7] E. Gull, A. J. Millis, A. I. Lichtenstein, A. N. Rubtsov, M. Troyer, P. Werner, Continuoustime monte carlo methods for quantum impurity models, Rev. Mod. Phys. 83 (2011) 349-404. doi:10.1103/RevModPhys.83.349

URL http://link.aps.org/doi/10.1103/ RevModPhys. 83.349

[8] K. S. D. Beach, R. J. Gooding, F. Marsiglio, Reliable padé analytical continuation method based on a high-accuracy symbolic computation algorithm, Phys. Rev. B 61 (2000) 5147-5157. doi:10.1103/ PhysRevB.61.5147

[9] G. A. Baker, P. Graves-Morris, Pad Approximants, 2nd Edition, Cambridge University Press, 1996, cambridge Books Online.

URL CB09780511530074

http://dx.doi.org/10.1017/

[10] X. Zhu, G. Zhu, A method for directly finding the denominator values of rational interpolants, J. Comput. Appl. Math. 148 (2) (2002) 341-348. doi: 10.1016/S0377-0427(02)00554-X.

[11] N. V. Prokof'ev, B. V. Svistunov, Spectral analysis by the method of consistent constraints, JETP Letters 97 (11) (2013) 649-653. doi:10.1134/ S002136401311009X

[12] A. W. Sandvik, Stochastic method for analytic continuation of quantum monte carlo data, Phys. Rev. B 57 (1998) 10287-10290. doi:10.1103/PhysRevB. 57.10287 .

[13] S. Fuchs, T. Pruschke, M. Jarrell, Analytic continuation of quantum monte carlo data by stochastic analytical inference, Phys. Rev. E 81 (2010) 056701. doi:10.1103/PhysRevE.81.056701.

URL http://link.aps.org/doi/10.1103/ PhysRevE.81.056701

[14] M. Jarrell, J. E. Gubernatis, Bayesian inference and the analytic continuation of imaginary-time quantum monte carlo data, Physics Reports 269 (3) (1996) 133-195.

[15] R. K. Bryan, Maximum entropy analysis of oversampled data problems, Eur Biophys J 18 (1990) 165-174.

[16] A. Albuquerque, F. Alet, P. Corboz, et al., The alps project release 1.3: Open-source software for strongly correlated systems, Journal of Magnetism and Magnetic Materials 310 (2, Part 2) (2007) 1187-1193.

URL http://dx.doi.org/10.1016/j.jmmm.2006. 10.304

[17] E. Gull, P. Werner, S. Fuchs, B. Surer, T. Pruschke, M. Troyer, Continuous-time quantum Monte Carlo impurity solvers, Comp. Phys. Comm. 182 (4) (2011) 1078-1082. doi:10.1016/j.cpc.2010.12.050.

[18] G. Mahan, Many-Particle Physics, Physics of Solids and Liquids, Springer US, 2000. doi:10.1007/ 978-1-4757-5714-9.

[19] C. E. Shannon, A mathematical theory of communication, Bell System Technical Journal 27 (1948) 379. doi:10.1002/j.1538-7305.1948.tb01338.x.

[20] S. F. Gull, J. Skilling, Maximum entropy method in image processing, Communications, Radar and Signal Processing, IEE Proceedings F 131 (6) (1984) 646659. doi:10.1049/ip-f-1:19840099

[21] D. W. Marquardt, An algorithm for least-squares estimation of nonlinear parameters, Journal of the Society for Industrial and Applied Mathematics 11 (2) (1963) 431-441. doi:10.1137/0111030 URL http://dx.doi .org/10.1137/0111030

[22] K. S. D. Beach, Identifying the maximum entropy method as a special limit of stochastic analytic continuation, arXiv:cond-mat (2004) 0403055. URL http: //arxiv .org/abs/cond-mat/0403055

[23] X. Wang, E. Gull, L. de' Medici, M. Capone, A. J. Millis, Antiferromagnetism and the gap of a mott insulator: Results from analytic continuation of the self-energy, Phys. Rev. B 80 (2009) 045101. doi: 10.1103/PhysRevB.80.045101.

[24] L. Boehnke, H. Hafermann, M. Ferrero, F. Lechermann, O. Parcollet, Orthogonal polynomial representation of imaginary-time green's functions, Phys. Rev. B 84 (2011) 075145. doi:10.1103/PhysRevB. 84.075145 .

[25] A. A. Kananenka, J. J. Phillips, D. Zgid, Efficient temperature-dependent greens functions methods for realistic systems: Compact grids for orthogonal polynomial transforms, Journal of Chemical Theory and Computation 12 (2) (2016) 564-571, pMID: 26735685. doi:10.1021/acs.jctc.5b00884. URL http://dx.doi.org/10.1021/acs.jctc. $5 \mathrm{b00884}$

[26] B. Gough, GNU Scientific Library Reference Manual - Third Edition, 3rd Edition, Network Theory Ltd., 2009. URL http://www.gnu.org/software/gsl/

[27] J. P. F. LeBlanc, A. E. Antipov, F. Becca, I. W. Bulik, G. K.-L. Chan, C.-M. Chung, Y. Deng, M. Ferrero, T. M. Henderson, C. A. Jiménez-Hoyos, E. Kozik, X.-W. Liu, A. J. Millis, N. V. Prokof'ev, M. Qin, G. E. Scuseria, H. Shi, B. V. Svistunov, L. F. Tocchio, I. S. Tupitsyn, S. R. White, S. Zhang, B.-X. Zheng, Z. Zhu, E. Gull, Solutions of the two-dimensional hubbard model: Benchmarks and results from a wide range of numerical algorithms, Phys. Rev. X 5 (2015) 041041. doi:10.1103/PhysRevX.5.041041.

[28] A. Georges, G. Kotliar, W. Krauth, M. J. Rozenberg, Dynamical mean-field theory of strongly correlated fermion systems and the limit of infinite dimensions, Rev. Mod. Phys. 68 (1996) 13-125. doi:10.1103/ RevModPhys.68.13

[29] A. Georges, W. Krauth, Physical properties of the half-filled hubbard model in infinite dimensions, Phys. Rev. B 48 (1993) 7167-7182. doi:10.1103/ PhysRevB.48.7167.

[30] T. Pruschke, D. L. Cox, M. Jarrell, Transport proper- 
ties of the infinite-dimensional hubbard model, EPL

(Europhysics Letters) 21 (5) (1993) 593.

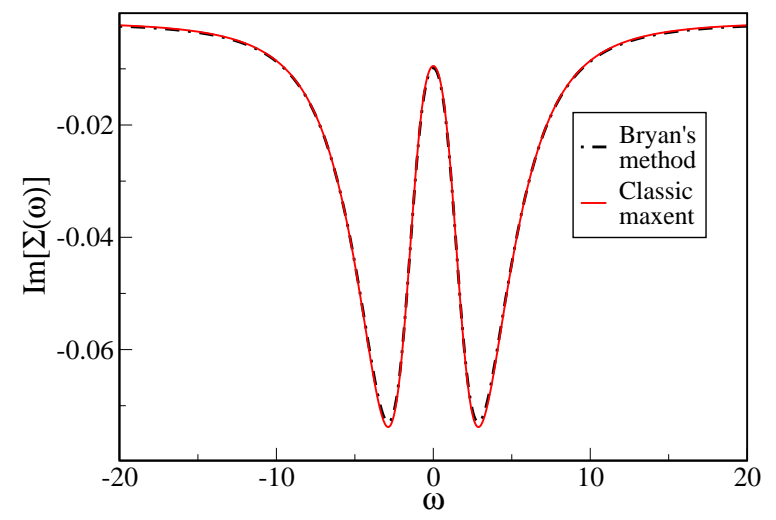

(a)

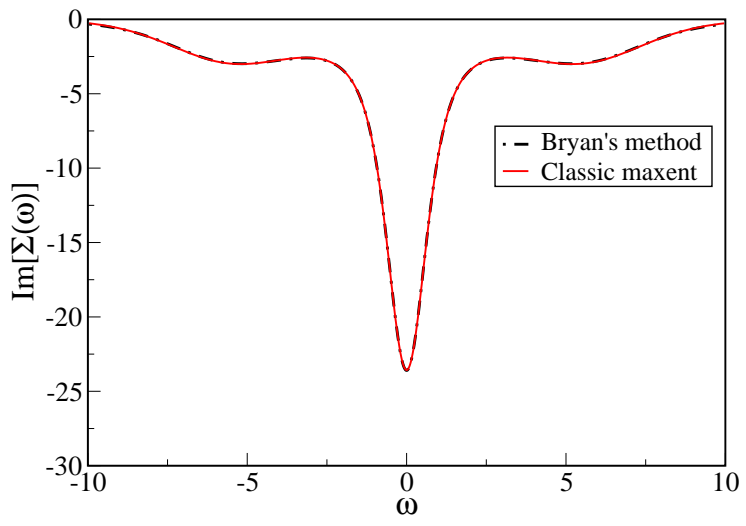

(b)

Figure 2: Maxent output of the imaginary part of the self-energy at $\beta t=2$ for: (a) $U / t=1$. (b) $U / t=10$. Black curves are from Bryan's method and red curves from historic Maxent 


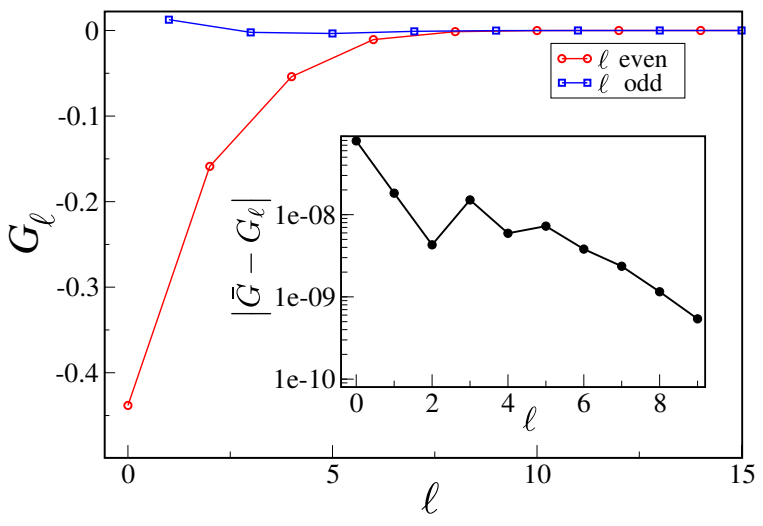

(a)

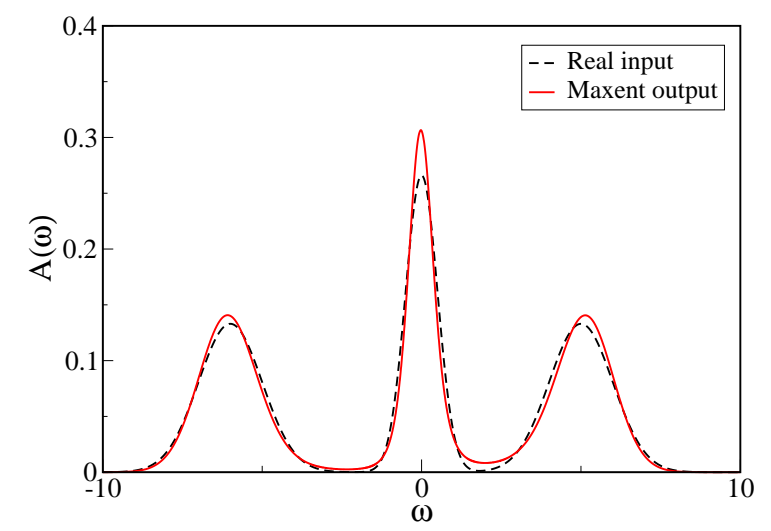

(b)

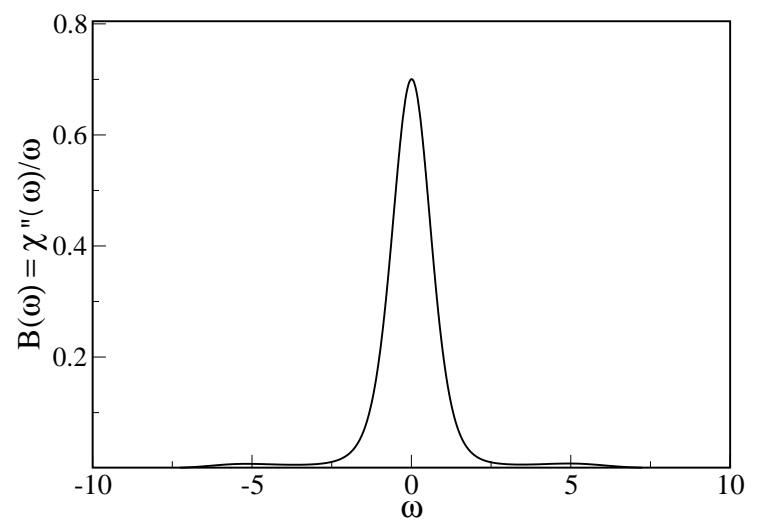

(a)

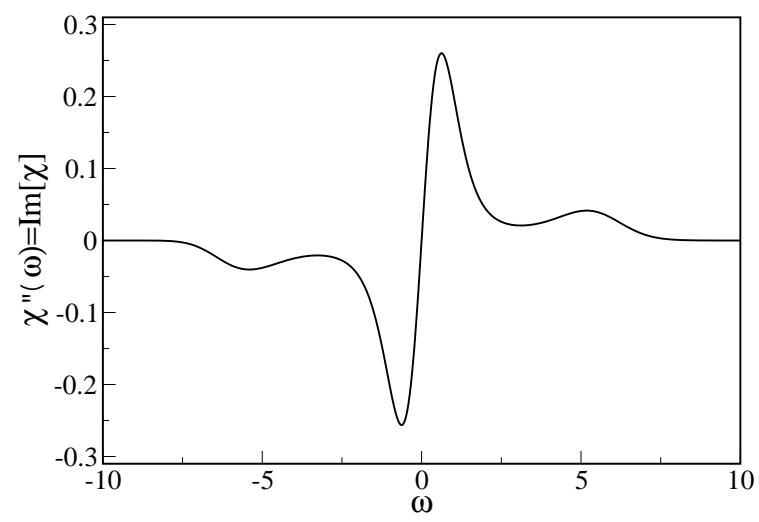

(b)

Figure 4: Maxent output for the magnetic susceptibility using bosonic Kernal. (a) Normalized function $B(\omega)=\chi^{\prime \prime}(\omega) / \omega$. (b) The imaginary part of the susceptibility $\chi^{\prime \prime}(\omega)$. 


\begin{tabular}{|c|c|c|}
\hline \multirow{2}{*}{ Name } & \multirow{2}{*}{ Expression } & Required Variables \\
\hline & & Default Variables \\
\hline \multicolumn{3}{|c|}{ Default Models $d(\omega)$} \\
\hline Flat & $1 /\left(\omega_{\max }-\omega_{\min }\right)$ & - \\
\hline \multirow{2}{*}{ Gaussian } & \multirow{2}{*}{$\frac{1}{\sqrt{2 \pi} \sigma} \exp \left[-\omega^{2} /\left(2 \sigma^{2}\right)\right]$} & $\sigma=\mathrm{SIGMA}$ \\
\hline & & - \\
\hline \multirow{2}{*}{ Double Gaussian } & \multirow{2}{*}{$\frac{1}{2}\left(\frac{1}{\sqrt{2 \pi} \sigma} e^{-\frac{(\omega-\text { SHIFT })^{2}}{2 \sigma^{2}}}+\frac{1}{\sqrt{2 \pi} \sigma} e^{-\frac{(\omega+\text { SHIFT })^{2}}{2 \sigma^{2}}}\right)$} & $\sigma=\mathrm{SIGMA}$ \\
\hline & & $\mathrm{SHIFT}=0$ \\
\hline \multirow{2}{*}{ Two Gaussians } & \multirow{2}{*}{$\frac{\text { NORM1 }}{\sqrt{2 \pi} \sigma 1} e^{-\frac{(\omega-\text { SHIFT1 })^{2}}{2 \sigma 1^{2}}}+\frac{(1-\text { NORM1 })}{\sqrt{2 \pi} \sigma 2} e^{-\frac{(\omega-\text { SHIFT2 })^{2}}{2 \sigma 2^{2}}}$} & SIGMA1, SIGMA2, SHIFT2 \\
\hline & & NORM1 $=0.5$, SHIFT $1=0$ \\
\hline \multirow{2}{*}{ Shifted Gaussian } & \multirow{2}{*}{$\frac{1}{\sqrt{2 \pi} \sigma} \exp \left[-(\omega-\mathrm{SHIFT})^{2} /\left(2 \sigma^{2}\right)\right]$} & $\sigma=\mathrm{SIGMA}$ \\
\hline & & $\mathrm{SHIFT}=0$ \\
\hline Lorentzian & $1 /\left(\pi \gamma\left[1+\left(\frac{\omega}{\gamma}\right)^{2}\right]\right)$ & $\gamma=$ GAMMA \\
\hline \multirow{2}{*}{ LinearRiseExpDecay } & \multirow{2}{*}{$\lambda^{2} \omega \exp [-\lambda \omega]$} & $\lambda=\mathrm{LAMBDA}$ \\
\hline & & - \\
\hline \multirow{2}{*}{ QuadraticRiseExpDecay } & \multirow{2}{*}{$\frac{\lambda^{3}}{2} \omega^{2} \exp [-\lambda \omega]$} & $\lambda=\mathrm{LAMBDA}$ \\
\hline & & - \\
\hline
\end{tabular}

Table 1: Default model $d(\omega)$ names and expressions that are available in Maxent. 


\begin{tabular}{|c|c|c|}
\hline \multirow{2}{*}{ Name } & \multirow{2}{*}{ Expression } & Required Variables \\
\hline & & Default Variables \\
\hline \multicolumn{3}{|c|}{ Grids } \\
\hline \multirow{2}{*}{ Lorentzian } & \multirow{2}{*}{$t_{i}=\tan \left[\pi \frac{i}{\text { NFREQ }}(1-2 \mathrm{CUT})+\mathrm{CUT}-0.5\right]$} & - \\
\hline & & $\mathrm{CUT}=0.01$ \\
\hline \multirow{2}{*}{ Half-Lorentzian } & \multirow{2}{*}{$t_{i}=\tan \left[\pi \frac{i+\mathrm{NFREQ}}{2 \mathrm{NFREQ}+1}(1-2 \mathrm{CUT})+\mathrm{CUT}-0.5\right]$} & - \\
\hline & & CUT $=0.01$ \\
\hline \multirow[t]{2}{*}{ Quadratic } & \multirow{2}{*}{$\Delta t=\frac{4(\mathrm{SPREAD}-1) \cdot\left[\left(\frac{i}{\mathrm{NFREQ}}\right)^{2}-\frac{i}{\mathrm{NFREQ}}\right]+\mathrm{SPREAD}}{\mathrm{NFREQ} /[3(\mathrm{NFREQ}-1)(\mathrm{NFREQ} \cdot(2+\mathrm{SPREAD})-4+\mathrm{SPREAD}]}$} & - \\
\hline & & $\mathrm{SPREAD}=4$ \\
\hline \multirow{2}{*}{$\log$} & \multirow{2}{*}{$t_{i} \sim 0.5 \pm$ LOG_MIN $\exp [i \cdot \log (0.5 /$ LOG_MIN $) /($ NFREQ $/ 2-1)]$} & - \\
\hline & & LOG_MIN $=0.0001$ \\
\hline Linear & $t_{i}=\frac{i}{\text { NFREQ }}$ & - \\
\hline
\end{tabular}

Table 2: Grid names and expressions that are available in Maxent. A real frequency grid is generated with $t_{i} \in[0,1]$ and then mapped to the range $\omega_{i} \in\left[\omega_{\min }, \omega_{\max }\right]$. The bounds for the frequency grid are controlled through the parameters OMEGA_MAX and OMEGA_MIN.

\begin{tabular}{|c|c|}
\hline name.out.avspec.dat & Spectral function using Bayesian Averaging - Bryan's method \\
\hline name.out.avspec_back.dat & The back-continued avspec spectrum \\
\hline name.out. chi2. dat & Estimated $\chi^{2}$ for each $\alpha$ value solution \\
\hline name.out. chispec.dat & Spectral function satisfying the best $\chi^{2}$ - historic maxent \\
\hline name out.chispec back. dat & The back-continued chispec spectrum \\
\hline name.out.fits.dat & Fits of each $\alpha$ value, see comments in file \\
\hline name.out. $\overline{\operatorname{maxspec}}$.dat & Spectral function with the highest probability - classic maxent \\
\hline name.out.maxspec_back.dat & The back-continued maxspec spectrum \\
\hline name.out.out.h5 & All output data in the hdf5 format \\
\hline name. out.prob. dat & The posterior probability of each $\alpha$ value \\
\hline name.out.spex. dat & All spectral functions produced; one for each \\
\hline
\end{tabular}

Table 5: Output files from Maxent given an input file name.param. The prefix name is replaced with the basename of the input file, or specified with the parameter BASENAME. 